\title{
Key role and potential of Industrial Internet of Things (IloT) in modern production monitoring applications
}

\author{
Jakub Pizoń ${ }^{1}$, Grzegorz Kłosowski ${ }^{1}$, and Jerzy Lipski ${ }^{1}$ \\ ${ }^{1}$ Lublin University of Technology, Faculty of Management, Department of Organisation of Enterprises, Nadbystrzycka 38, 20-618 \\ Lublin, Poland
}

\begin{abstract}
The following paper presents a key role and potential of Industrial Internet of Things (IIoT) in industrial applications as a solution for monitoring and maintaining manufacturing assets. IIoT is particularly important due to progressing computerisation of hardware resources leading to development of a virtualised model of autonomous real-time production management. Adequately article presents case study of IIoT use in production environment - both methodical and analytic approach is presented.
\end{abstract}

\section{Introduction}

Progress in collection and analysis of data from devices enables transferring predictive maintenance[1] onto a higher level. By connecting sensors to machines and streaming the sensor readings in real time to a data processing and analysis platform, it is possible to capture operational metrics of equipment[2]. These metrics can be stored in a time series database and employed to search for indicators that could be applied in equipment failure prediction. Ultimately this prediction model could use live data from production facilities. The key to functioning of such solutions is the use of modern advances in the IT. These solutions are based on a paradigm of the Industrial Internet of Things and developed accordingly to its determinants [3]. Thanks to the platform for device management and data analysis, it is possible to implement necessary steps. Using appropriate technologies compliant with the paradigm, it is possible to propose a technological stack that not only meets the requirements of data collection and processing, but also allows for its extension from tens and hundreds of devices to tens and hundreds of thousands of devices. [4]

In recent decades, manufacturers and suppliers of goods and services have improved the quality of their organisations using innovative technologies [5]. That progress would not be possible without fundamental transformation and evolution of industries towards full digitisation and intelligence of production processes to ensure high efficiency. The achieving of these goals requires implementation of new technologies for the automation of industrial processes. These concepts are the pillars of the fourth industrial revolution called "Industry 4.0" [6]. The fourth industrial revolution was invented by Klaus Schwab, the founder and CEO of the World Economic Forum. This term describes a world in which people move between digital domains and offline reality using combined technology to facilitate and manage their lives. This term has been popularised and spread in literature since the Hanover Conference in 2011 [7].

Data acquisition is the key to 4.0 Industry solutions. Therefore, the monitoring of production, processes and machines is an underlying element of any application of the Industry 4.0 assumptions. Without data regarding machine wear and tear or errors, it is not possible to process information on machine operation.

The smart industry as a fourth generation is referred to as Industry 4.0 and is based on cyber-physical systems which provide the internet connection. The concept of Industry 4.0 with the internet of things addresses great expectations of industries providing solutions in several aspects. Nowadays many countries introduce high-tech strategies focusing their research and innovation policy on selected forward-looking projects related to scientific and technological developments [8].

Moreover, implementation of Industry 4.0 is a complex, multidimensional task that involves formal, technical and economic changes in the company's operations:

- preparation of qualified personnel capable of applying digital and intellectual production technologies in a field of exploitation of system's cyber-physical components,

- $\quad$ preparation of standards in a field of machines and design of new generation elements and amendment of laws, adjusting them to the conditions of digital economy, which regulates an activity of the company [9].

Currently, flexible MRP II and ERP systems based on CIM are dominated by batch processing. The dominant structure is a hierarchical structure of information transmission, which improves organisation of the entire production process, however making it difficult to take

* Corresponding author: jakub.pizon@ @ollub.edu.pl 
quick operational decisions from a production level (floor shop) [10]. This provides space for optimisation and intensification of production. In this respect, modern technologies are implemented, such as edge and fog computing [11]. The basis for an application of these technologies is an ability to quickly receive and transmit data of the monitored process, machine or state.

\section{Monitoring and maintenance}

Monitoring of production machines and their predictive maintenance is responsibility of maintenance department. Maintenance costs, depending on a size of the machinery and the number of machines incorporated in the manufacturing process, constitute a fixed production cost. As a fixed cost, it increases the level of funds frozen by the company in production, and therefore the investment and R\&D opportunities are reduced.

Monitoring is laborious and requires considerable amount of time and attention from the personnel. Moreover, gathering and processing of collected data is not easy because of reasons such as: errors, lack of data, lack of continuity of data, machine failures, line layout change, outdated data [12]. What is more, due to a large variety of machines, it is hard or even impossible to unify a single monitoring function or application. Moreover, strictly environmental factors like temperature, humidity, vibrations etc. have also a continuous effect on the machines monitored and maintained [13]. Therefore, the process of change is ongoing and requires constant monitoring.

\subsection{Problems with IT monitoring systems}

With help comes IT monitoring systems. However, certain problems may also arise in this respect, e.g.:

1. Vendor lock-in - situation where the customer is dependent on the supplier's products to such an extent that they cannot change the supplier without incurring the costs of change.

2. Monitoring system maintenance cost that is related to IT cost of infrastructure (servers, connections, updates).

3. Local on-premise solutions - lack of scaling and flexibility.

4. Special monitoring equipment purchase - costs, which do not translate into a rapid increase in profits.

5. Fast changing technology - risk of not being updated or supported by the manufacturer.

6. Closed systems - system do not support integration or extension according to emerging needs.

Presented points show reasons why companies, and particularly those without maintenance departments monitoring and maintenance of machinery, are either overlooked or severely neglected. The dominant belief is that if it works, there is no need for maintenance. The machines run until failure occurs. Nevertheless, what such companies appear to be oblivious to is that elimination of defect can greatly exceed the costs of proper maintenance. It can be assumed that this belief prevails in small and medium-sized enterprises operating in any perspective of time [14]. The situation is significantly improved in large companies; however, negligence occurs everywhere.
Minimising fixed costs is one of the areas where there is a great need to search for new effective solutions.

Moreover, one of the key objectives of monitoring and maintenance systems is to replace human reactions in critical and emergency states. Given high complexity of modern manufacturing systems and many signals reaching an operator, an effect of "information overload" is to be expected. As a result, the operator may take incorrect actions resulting in further failures [15].

In addition, many of the phenomena that accompany technological processes take place at such a high speed that it is impossible to observe them without special autonomous and visualising systems.

\subsection{Process}

Developing a set of relevant concepts plays a vital role in implementation of monitoring and predictive maintenance functions of machines. The set of concepts is then used to define the key phases of the planned control process.

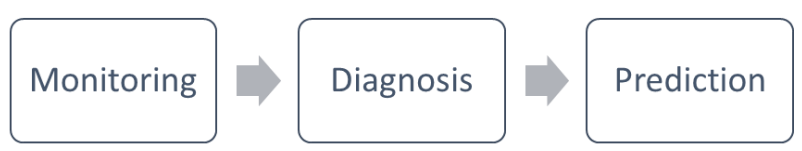

Fig. 1. Monitoring and predictive maintenance process.

Monitoring is carried out when a machine is tracked (monitored) using available techniques and devices. Condition monitoring can be continuous or periodic which means that it can be conducted from time to time or preferably at a constant frequency.

Diagnostics, on the other hand, is an ability to correctly interpret information obtained from monitoring (the ability to diagnose) and to draw appropriate conclusions as to whether the machine is working correctly or whether their failure has occurred. In the case of simple machines, symptoms of individual failure are relatively easy to detect for an experienced diagnostician. In the case of more complex structures, however, it may not be entirely obvious anymore [16].

From the manufacturing system viewpoint, the basic task of diagnostics is to maintain continuity of the manufacturing process. However, it should be remembered that it depends on many factors. The role of additional systems supporting the basic process is:

- providing information in an appropriate form for individual workstations and technological machines, as well as for transport and storage systems,

- ensuring a supply of energy with the desired parameters to sub-basic and auxiliary processes,

- $\quad$ ensuring a flow of materials with fixed parameters and over a specified period [15].

Forecasting, on the other hand, concentrates all activities aimed at estimating what will happen to a machine in the future (near or far). The key here is to determine how long the machine will still be able to handle data in its hope for proper functioning [16]. In this case, a final answer is obtained by combining insights from several different sources, such as a speed at which failure occurs, real-time observations (present state), 
complex mathematical models based on probabilistic calculus, and finally special tables that contain a history of similar events and help predict what may happen.

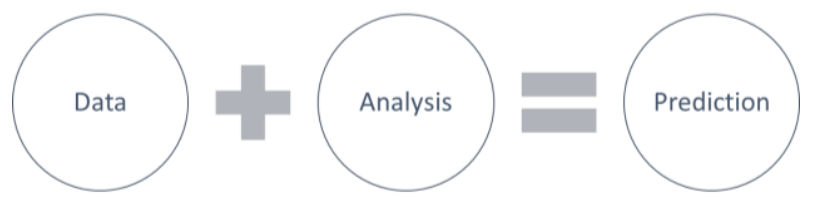

Fig. 2. Prediction action equation.

\section{IloT production monitoring}

The basis for Industrial Internet of Things (IIoT) is the Internet of Things (IoT). IoT is an information network of physical objects (sensors, machines, cars, buildings and other objects) that allows these objects to interact and cooperate to achieve common goals [17]. IIoT has emerged as a general concept for the use of the Internet of Things in the industrial sector. In fact, this is generalisation of Industry 4.0, which seems to focus more on efficiency of industrial processes and relates to an expected economic impact. This means that IIoT leads to Industry 4.0. IIoT's vision covers all aspects of industrial operations, focusing not only on process efficiency, but also on asset management, maintenance, etc. The vision of IIoT covers all aspects of industrial operations.

The key role of industrial IoT in modern monitoring of production machines and their predictive maintenance lies in their ability to aggregate data in real time [18]. This is data of physical dimensions of machines, products, but simultaneously of an environment in which a device is located.

This capability is provided by CPS (Cyber-Physical Systems), which create the Industrial Internet of Things. Cyber-Physical Systems consist in close integration of a computational layer and physical processes. They most often occur in a form of embedded computers and networks for monitoring and controlling physical processes that operate in feedback, where physical processes affect calculations and vice versa [19]. CPS solutions are used to implement a concept of Cyber Manufacturing Systems (CMS). CMS is a transformative concept that translates data from interconnected systems into predictive and prescriptive operations to ensure high performance [20].

The CMS performs its function based on data derived from the CPS in accordance with IIoT by means of stream data processing. Lambda architecture of data processing, which allows to analyse production data online, is most frequently used for this purpose [21]. It should be noted, however, that every optimisation in this area translates into economic effect as it reduces a percentage of production in progress, thus reducing production costs incurred by the company. In the context of production, Cyber Manufacturing Systems (CMS) and IIoT denote their relevant industrial equivalents of CPS and IoT.

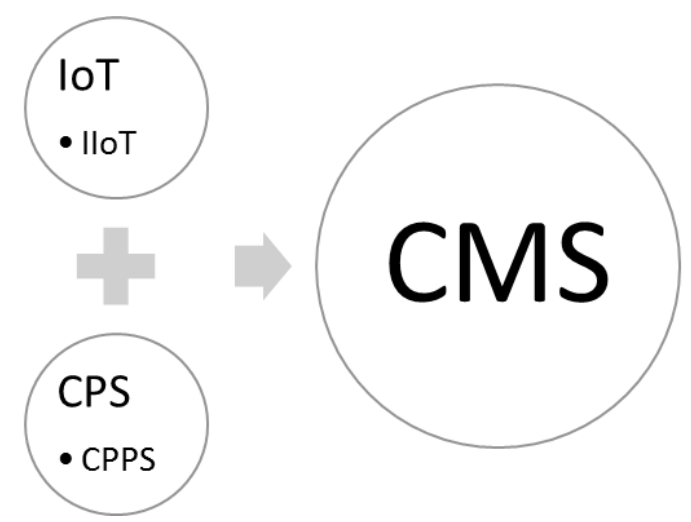

Fig. 3. CMS composition.

Therefore, in general, CPPS and IIoT are not individual, closed loop architectural and conceptual technologies. These technologies provide an interdisciplinary connection between production, information technology, mechatronics, communication technology and ergonomics. Therefore, to understand, predict and optimise the behaviour of systems, it is common to build models that can simulate the dynamics of the represented system. It is particularly important that IIoT and CMS are used mainly to solve dedicated problems [22]. This means that manufacturers provide software, sensors compliant with the IIoT and CMS, but implementation and addressing of monitoring and maintenance needs is crucial. Therefore, the context is more important, i.e. what is to be monitored and why than how monitoring is to take place. Determination of a context is crucial to implementation of a modern IIoT and CMS solution.

In addition to explicit data of the process, which result from the context of taken actions, implicit data attract significant attention. They represent a status of process environment. These signals can affect the condition of a machine or an auxiliary process. The data are typically omitted (unless they are process-relevant) because of their perceived low relevance to manufacturing.

On the other hand, omitted data may contain additional information of considerable importance to the basic production process as well as for the auxiliary processes. Therefore, this is not only the machine but also the operator, whose decisions have bearing on the condition of machines that can be affected by data. This in turn may translate into the entire process and its functioning.

Moreover, as non-confidential data are not used directly for orchestrating machines and devices, an effect of their aggregation is not immediate. This datum is collected in a form of time series, therefore illustrating changes over a longer period. This makes it possible to identify disturbances and provides evidence for possible complaints about machines and equipment [23]. 


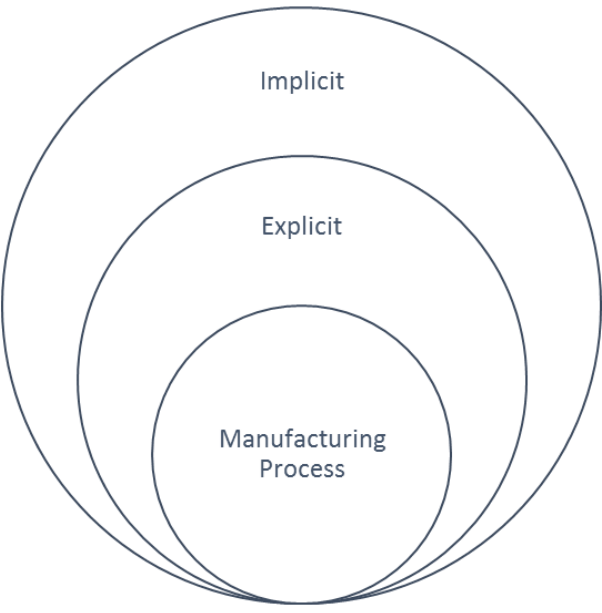

Fig. 4. Manufacturing data acquisition areas.

\section{Case study}

Since man began to build machinery for industrial applications, especially since engines started to power machines and production lines, problems with limiting and isolating vibrations have become essential.

Industrial vibration analysis is a tool used to identify, predict and prevent failure in mechanical machinery. Vibration analysis machine implementation will improve machine reliability and lead to its better performance and reduced downtime to fix mechanical or electrical faults.

Analysis and monitoring can be presented as a set of consistent steps to detect anomalies [24]. The analysis is not performed on raw but processed data, which allows to design appropriate data. Vibration analysis is carried out in an identical manner throughout the industrial world to detect machine failure, plan machine repairs and store the machine running if possible without failure.

With modern data collection and plant analysis solutions, we can take industrial predictive maintenance to the next level [25]. By connecting sensors to devices and sending readings from a streaming sensor to a data platform, we can capture hardware operating indicators. We can store these data in a timeseries database, study indicators that would help us predict equipment failure, and ultimately use this predictive model on live data from production facilities [26].

\subsection{Configuration}

The environment for data acquisition is configured in three steps. First, it is necessary to create a space that will be a place of creating virtual devices. Then connect a physical device to the platform solution. The next step is to analyse and visualise obtained data.

The above steps will be performed using DeviceHive platform for managing IoT and Apache Zeppelin devices operating at the top of Apache Spark stack programmed from the Scala language level.

\subsection{Implementation}

To present a strategy, an acceleration sensor (accelerometer) working in three axes was connected. By means of its readings, a failure of equipment is to be observed. Accelerator readings will be used to detect machine malfunction.

This peripheral receives an accelerometer signal and sends it to the DeviceHive playback. The platform subsequently sends data as a stream to the Apache Kafka information bus as csv file.

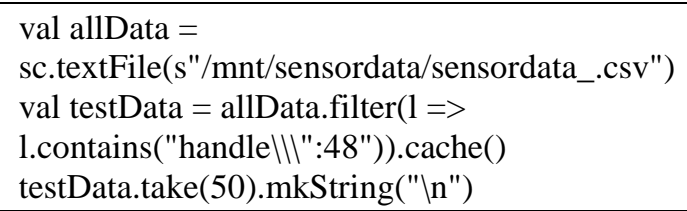

Fig. 5. Retrieving data.

From an analytical point of view, several adjustments still need to be made. Above all, instead of three axes, it is safer to use the Euclidean distance to aggregate data into a single measurement. For easier processing, the data sets will be saved into a short form consisting of a time stamp, a notification tag and a distance tag. At this point, the question arises of how to detect vibrations effectively. Therefore, a measure of variance is used instead of an absolute measure.

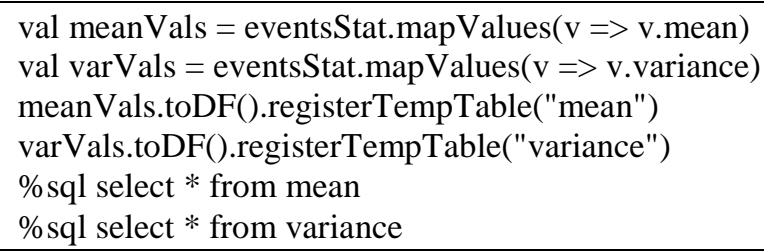

Fig. 6. Variance calculation.

This measure makes it possible to see that a variant increase and decreases with the start of the machine operation as the work is finished. Indeed, may be clearly seen that the variation increases when the fan started to vibrate. This model can now be tested in a real-time data environment.

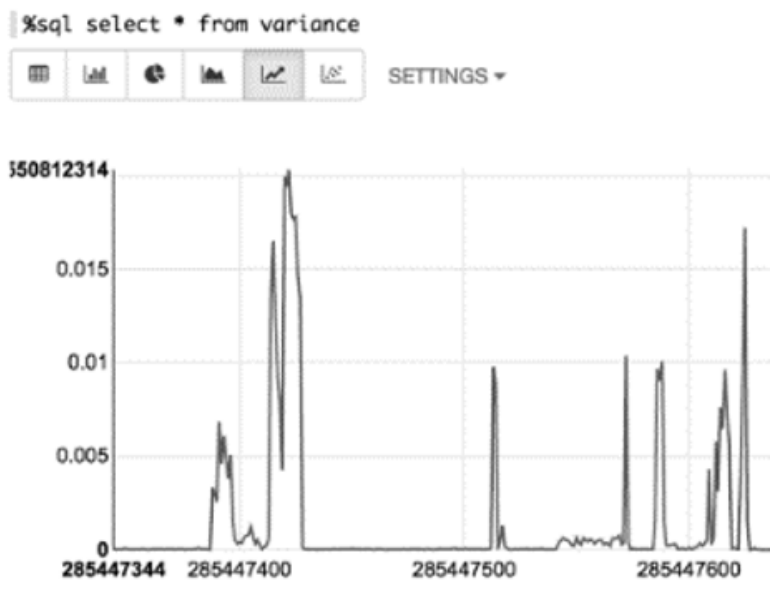

Fig. 7. Variance graph.

Having defined a model and having set a threshold, we can now run our model in live data stream using Spark Streaming, which uses the same concepts as Spark. First, we need to add third-party parsing libraries JSON and 
Kafka streaming. We call up the results using the SQL query from the Apache Zeppelin notebook.

\%sql select deviceGuid, value, timestamp from notifications where value $>\$\{$ threshold $=0.2\}$

Fig. 8. Preventive actions query.

The strategy indicates that IoT tools such as DeviceHive and Spark can capture and explore device data that can lead to predictive maintenance models being built and used on a large scale in the production environment.

The DeviceHive platform provides tools for quick capturing of hardware operating parameters and transferring them to the data platform. Apache Spark and Apache Zeppelin provide the means to explore, prototype and visualise data.

\section{Conclusion}

IIoT revolutionises production, allowing manufacturing business to acquire and access much more data at considerably higher speeds and much more efficiently than ever before. This will be particularly the case for monitoring and predictive maintenance of production machinery. Predominantly since IoT solutions are tremendously versatile in the production company's space.

What is important is that this type of solution is not a trivial undertaking. It requires interdisciplinary knowledge so that a CMS solution based on IIoT can perform the right tasks. The level of available tools allows creating flexible monitoring systems aggregating data both horizontally and, in a report, (vertically).

Moreover, as it was presented in case study it can be based on readymade IoT solutions. Therefore, engineers and constructors can be focused more on issues of production preparation and a little bit less on development of tools. Therefore, in IIoT solutions, the context and expected value of implemented activities becomes crucial. It is particularly important that due to progressing computerisation of hardware resources we are approaching a fully virtualised model of autonomous realtime production management.

Therefore, it is reasonable to state that during the construction and modernisation of production systems it is necessary to consider the organisation of their elements, both in technical and logistic terms, as well as IT solutions, aimed at minimising human activity within the operational and managerial part of the system, creating modern autonomous production processes.

\section{References}

1. E. Sezer, D. Romero, F. Guedea, M. Macchi, C. Emmanouilidis, IEEE International Conference on Engineering, Technology and Innovation (ICE/ITMC), 1 (2018)

2. B. Luetkehoff, M. Blum, M. Schroeter. IFIP AICT 534 (2018)
3. J. Lee, H. Davari, J. Singh, V. Pandhare, MFGLET, in press, (2018)

4. J. Lee, H. Davari, J. Singh, V. Pandhare, MFGLET, in press, (2018)

5. M. Flores, D. Maklin, M. Golob, A. Al-Ashaab, C. Tucci, Collaborative Networks of Cognitive Systems, (19th IFIP WG 5.5 Working Conference on Virtual Enterprises, PRO-VE 2018, Cardiff, UK, September 17-19, 2018, Proceedings 2018)

6. D. Bittighofer, M. Dust, A. Irslinger, M. Liebich, L. Martin, IEEE International Conference on Engineering, Technology and Innovation (ICE/ITMC) 1 (2018)

7. A. Petrillo, D. Felice, R. Cioffi, F. Zomparelli, Digital Transformation in Smart Manufacturing (IntechOpen 1, 2018)

8. D. Trotta, P. Garengo, 7th International Conference on Industrial Technology and Management 113 (2018)

9. S. P. Dhal, P. Chowdhury, S. Shaw, International Journal of Computer Science and Mobile Applications, National Conference on "The Things Services and Applications of Internet of Things" (Gandhi Institute for Education and Technology (GIET) Baniatangi 23-24 March, 85, 2018)

10. A. Gurjanov, D. Zakoldaev, A. Shukalov, I. Zharinov, IOP Conf. Series: Materials Science and Engineering, 327 (2018)

11. K. Fettig, T. Gačić, A. Köskal, A. Kühn, F. Stuber, 2018 IEEE International Conference on Engineering, Technology and Innovation (ICE/ITMC) 1 (2018)

12. N. Verba, K. Chao, J. Lewandowski, N. Shah, A. James, F. Tian, FGCS 91, 48 (2019)

13. D. Libes, S. Shin, J. Woo, IEEE International Conference on Big Data (Big Data), 68 (2015)

14. E. Kosicka, E. Kozłowski, D. Mazurkiewicz, Advances in Intelligent Systems and Computing 637, 54 (2018)

15. Ł. Sobaszek, A. Gola, A. Świć, Predictive scheduling as a part of intelligent job scheduling system, Advances in Intelligent Systems and Computing 637, 358 (2018)

16. J. Lipski, Diagnostyka procesów wytwarzania (Biblioteka Politechniki Lubelskiej Ośrodek ds. Wydawnictw i Biblioteki Cyfrowej, 2013) (in Polish)

17. R. Knosala, Inżynieria produkcji. Kompendium wiedzy (Polskie Wydawnictwo Ekonomiczne, 2017) (in Polish)

18. S. Jeschke, C. Brecher, T. Meisen, D. Özdemir, T. Eschert, Cyber Manufacturing Systems, 3 (2017)

19. O. Senvar, E. Akkartal, IJIBM 10, 50 (2018)

20. E. Lee, Cyber Physical Systems: Design Challenges (11th IEEE International Symposium on Object and Component-Oriented Real-Time Distributed Computing (ISORC) 2008)

21. J. Lee, B. Bagheri, C. Jin, Manuf. Let. 8, 1 (2016) 
22. S. Athira, J. Smitha; IJARIT Journal 4, 306 (2018)

23. J. Pizoń, T. Cieplak, Zeszyt Naukowy.pl/Wyższa Szkoła Zarządzania i Bankowości w Krakowie 1 (2017) (in Polish)

24. D. Mourtzis, E. Vlachou, N. Milas, Procedia CIRP, 55, 290 (2016)
25. N. Caithness, D. Wallom, arXiv:1804.02998, (2018)

26. T. Cieplak, P. Muryjas, Introduction to Basic Integration of Measurement Devices with Cloud Computing Services, AMM 791, 34 (2015)

27. C. Liu, J. Ding, A. J. Toprac, T. Chai, KAIS 41, 401 (2014). 